\title{
Towards real-time identification of cosmic rays with LOw- Frequency ARray radio antennas
}

\author{
Antonio Bonardi ${ }^{1, \star}$, Stijn Buitink ${ }^{2}$, Arthur Corstanje ${ }^{1}$, J. Emilio Enriquez ${ }^{1}$, Heino Falcke ${ }^{1,3,4}$, Jörg \\ R. Hörandel ${ }^{1,4}$, Pragati Mitra ${ }^{2}$, Katie Mulrey ${ }^{2}$, Anna Nelles ${ }^{5}$, Jörg Paul Rachen ${ }^{1}$, Laura Rossetto ${ }^{1}$, \\ Pim Schellart ${ }^{1}$, Olaf Scholten ${ }^{6,7}$, Satyendra Thoudam ${ }^{1}$, Gia Trinh ${ }^{6,7}$, Sander ter Veen ${ }^{2}$, and Tobias \\ Winchen ${ }^{2}$ \\ ${ }^{1}$ Department of Astrophysics/IMAPP, Radboud University, P.O. Box 9010, 6500 GL Nijmegen (the Nether- \\ lands) \\ ${ }^{2}$ Vrije Universiteit Brussel, Pleinlaan 2, 1050 Brussels (Belgium) \\ ${ }^{3}$ Astron, Oude Hoogeveensedijk 4, 7991 PD Dwingeloo (The Netherlands) \\ ${ }^{4}$ Nikhef, Science Park 105, 1098 XG Amsterdam (The Netherlands) \\ ${ }^{5}$ University of California Irvine, Irvine, CA 92697, (USA) \\ ${ }^{6} \mathrm{KVI}$-CART, Zernikelaan 25, 9747 AA Groningen (The Netherlands) \\ ${ }^{7}$ University of Groningen, Zernikelaan 25, 9747 AA Groningen (The Netherlands)
}

\begin{abstract}
Cosmic rays entering the Earth's atmosphere produce Extensive Air Showers, which emit a radio signal through Geo-magnetic radiation and Askaryan emission. At the present time, one of the biggest challenges for assessing the Radio detection as a valuable technique for Cosmic-ray observation is to identify in real-time the very short (less than $100 \mathrm{~ns}$ ) radio signals over the background noise. In this work, we present the latest updates on the real-time identification of radio signals from Extensive Air Showers by using the data from LOFAR Low Band Antenna stations, which are sensitive in the 30-80 MHz region.
\end{abstract}

\section{Introduction}

When a Cosmic Ray enters the Earth's atmosphere, it starts an Extensive Air Shower (hereafter "EAS") of secondary particles, which emits a radio signal, through Geo-magnetic radiation and Askaryan emission.

It has been recently proven that the primary particle properties, as arrival direction, energy, and mass composition, can be reconstructed by ground-based radio detector arrays [1],[2],[3] with an accuracy similar, if not even better, than current Cosmic-ray experiments. The big advantage of the Radio-detection technique is its very high duty cycle (virtually up to $100 \%$ ) and its construction and operational costs, which are considerably smaller compared to current experiments based on Fluorescence-detection technique or secondary particles detection. On the other hand, due to the very short duration of the radio signal (less than $100 \mathrm{~ns}$ ) and the overwhelming background noise of Radio-Frequency Interferences (hereafter "RFI"), the direct detection of EAS with Radio detectors

^e-mail: a.bonardi@astro.ru.nl 


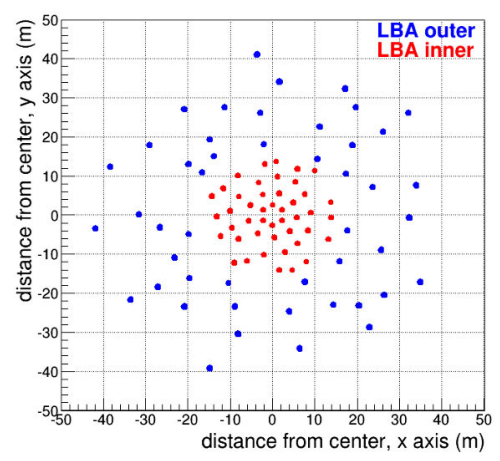

Figure 1. Antenna location in one LOFAR station for LBA inner and LBA outer configuration

only has been very difficult so far. As consequence, particle detectors are usually used in combination with Radio detectors as trigger for the Radio data acquisition, but this procedure sets currently a strong limitation to the exploitation of the Radio detection technique.

The multipurpose LOw-Frequency ARray (LOFAR)[4] is currently the best-suited experiment for developing a realtime Radio-detection algorithm for EASs, thanks to the external trigger provided by the LORA[5] particle detector array and to the very large number of omnidirectional Low-frequency Band Antennas (hereafter "LBA"), each of them equipped with two polarization arms NE-SW and NO-SE oriented and operational in the $30-80 \mathrm{MHz}$ frequency band. LBAs are clustered in units of 96 antennas, each of them called "station". The highest density of stations is the innermost region which is located nearby Exloo (the Netherlands), while remote stations are spread all around Central and Northern Europe. During standard astronomical observation, on each station only half of LBAs are active in the so-called "LBA inner" or "LBA outer" configuration, as shown in Fig. (1). When LORA provides a trigger, the time series $\left(\sim 2.2 \mathrm{~ms}\right.$ length, $\left.200 \mathrm{MSample} \mathrm{s}^{-1}\right)$ of each active antenna polarization is downloaded for further offline analysis. At the present time, just the innermost region is covered by the LORA array, significantly affecting the LOFAR Cosmic-ray detection at energies above $10^{18} \mathrm{eV}$, where a larger covered area is needed.

\section{The Radio self-trigger algorithm}

The developed algorithm consists of two steps: in the first step Radio pulses are detected ("selection phase"), while in the second one RFI-candidates are rejected ("RFI rejection phase").

During the selection phase, the radio signal of each antenna along both polarizations is compared with its baseline fluctuation: when on a certain antenna the signal of one polarization is above the given threshold, the antenna is considered as "fired" along that polarization. A station is considered "triggered" if at least 24 antennas are fired along the same polarization within a coincidence time of $20 \mathrm{~ns}$ (in the LBA inner configuration) or $30 \mathrm{~ns}$ (in the LBA outer configuration).

The RFI rejection phase is based on a multi-criteria approach, consisting of evaluating the radio pulse duration, its frequency spectrum, its timing and its arrival zenith angle. Radio pulses satisfying any of the following conditions are threated as RFI and rejected:

- pulse duration: the time window where 3 or more antennas are fired (within the coincidence time) is larger than $75 \mathrm{~ns}$ (LBA inner) or $300 \mathrm{~ns}$ (LBA outer); 
Table 1. Trigger performances for LBA inner configuration

\begin{tabular}{cccc}
\hline Threshold Level & CR triggers & rejected CR triggers & RFI triggers after rejection \\
\hline 3 RMS & 395 & $65 \%$ & 0 \\
4 RMS & 246 & $36 \%$ & 0 \\
5 RMS & 168 & $27 \%$ & 0 \\
10 RMS & 51 & $35 \%$ & 0 \\
\hline
\end{tabular}

Table 2. Trigger performances for LBA outer configuration

\begin{tabular}{cccc}
\hline Threshold Level & CR triggers & rejected CR triggers & RFI triggers after rejection \\
\hline 3 RMS & 1536 & $25 \%$ & 4 \\
4 RMS & 899 & $21 \%$ & 2 \\
5 RMS & 598 & $22 \%$ & 2 \\
10 RMS & 196 & $24 \%$ & 0 \\
\hline
\end{tabular}

- frequency spectrum: the ratio between the station mean pulse intensity in the $30-45 \mathrm{MHz}$ frequency band and in the $45-70 \mathrm{MHz}$ band is smaller than 1 or larger than 2;

- timing: the pulse occurs less than $5 \mu \mathrm{s}$ before/after from the next/previous triggered pulse;

- arrival direction: its reconstructed arrival zenith angle is larger than $60^{\circ}$ (only used with the LBA inner configuration).

\section{Analysis and Results}

For this study we considered the raw-data from the 4081 recorded events with LBA outer configuration and the 634 events with LBA inner configuration. All the considered events have time, energy, position, and arrival direction successfully reconstructed by LORA, and have at least one LOFAR station with all the antenna channels properly working. Stations with one or more missing read-out channels have been removed.

In the selection criterion four different threshold levels have been considered for the antenna "firing" condition, equal to 3, 4, 5, and 10 times the baseline RMS. The detected radio pulses are considered originated by the same EAS detected by LORA, if they occur within $5 \mu \mathrm{s}$ before and $12.5 \mu \mathrm{s}$ after the LORA time stamp. Any pulse falling outside this time window is considered as RFI pulse.

The results obtained for LBA inner and LBA outer are summarized in Table 1 and Table 2

For both LBA inner and LBA outer, the primary particle energy distributions of all the analyzed events and of the events generating a trigger in at least one station is shown in Fig. (2). The distributions of distance from the EAS core position of all the available stations and of the triggered ones are shown in Fig. (3) . Fig. (2) and Fig. (3) show that for LOFAR stations the energy threshold increases and the effective collecting area slightly decreases by increasing the trigger threshold level.

\section{Conclusions and Outlook}

For both LBA inner and LBA outer, we obtained a good trigger efficiency together with a very good RFI rejection efficiency, especially for primary energy above $2 \cdot 10^{17} \mathrm{eV}$. If we exclude data acquired in not fair-weather conditions, only one RFI pulse passed our selection and rejection criteria. This was found with 3 RMS threshold level and LBA outer configuration. 

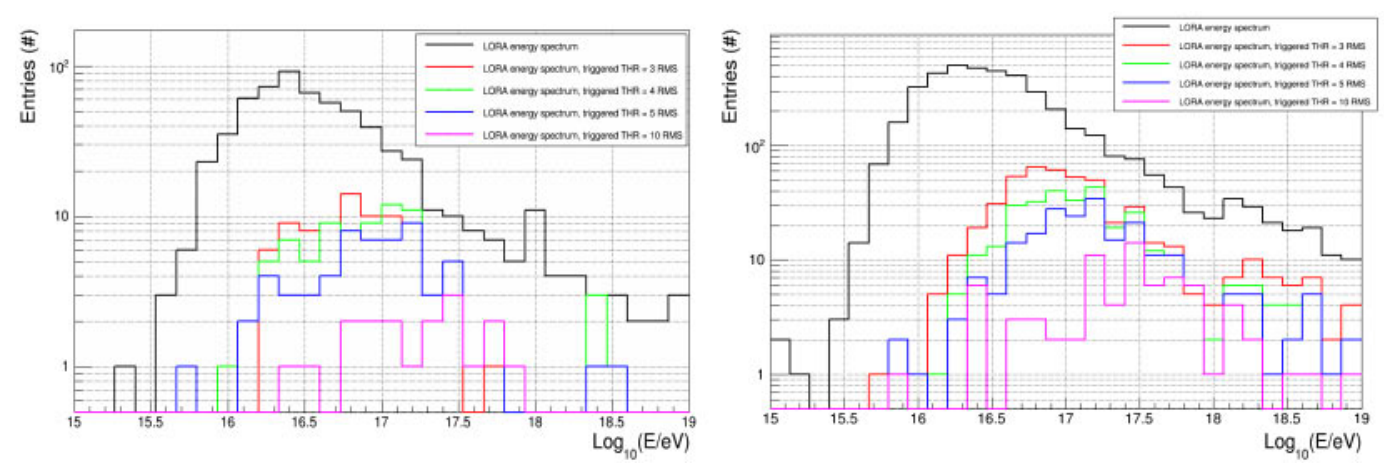

Figure 2. Primary particle energy distributions for LBA inner configuration (left) and LBA outer configuration (right), as reconstructed by LORA. The distribution of the whole set of events is shown together with the distribution of triggered events at the trigger threshold equal to 3, 4, 5, and 10 RMS.
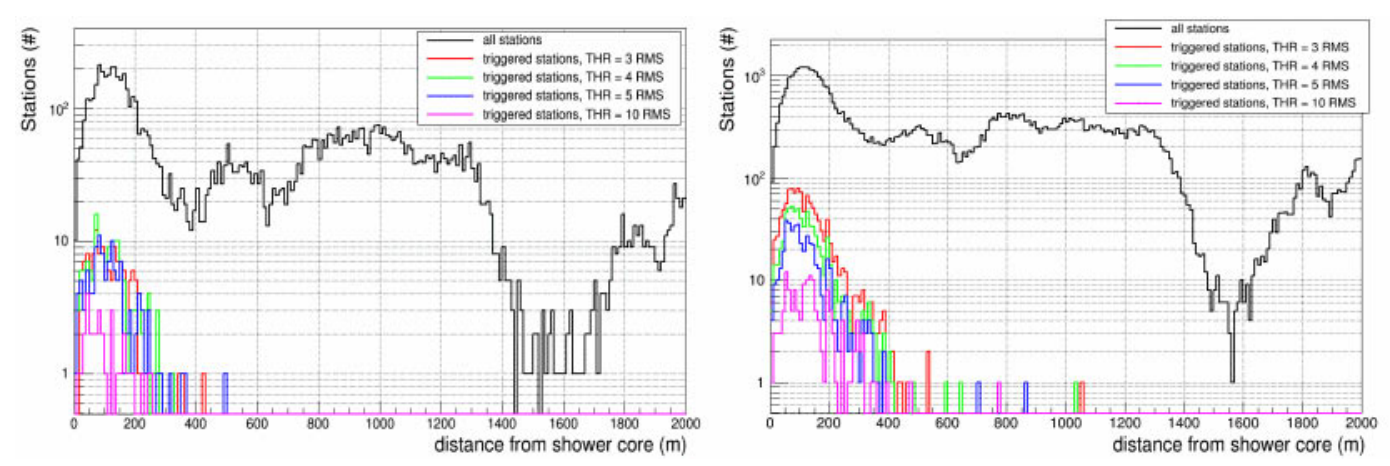

Figure 3. Station distance distribution from the LORA reconstructed EAS core position for LBA inner configuration (left) and LBA outer configuration (right). The distribution of all the available stations is shown together with the distributions of the only triggered stations at the 4 threshold levels.

On the other hand, the available statistics is not enough ( $\sim 9 \mathrm{~s}$ of independent live-time and $\sim 119 \mathrm{~s}$ total live-time for LBA outer, $\sim 2 \mathrm{~s}$ and $\sim 22 \mathrm{~s}$ for LBA inner) for concluding that the desired RFI rejection efficiency has been achieved. Therefore, low threshold acquisitions are planned to be performed on one LOFAR test station for increasing the available statistics. Once the RFI trigger rate will be found smaller than 1 per hour per station, the following step will be the implementation of the self-trigger algorithm to the LOFAR remote stations, so to boost up the cosmic-ray collecting area.

\section{References}

[1] Corstanje A. et al. Astropart. Phys. 61, 22 (2014)

[2] Nelles A. et al. JCAP 05, 18 (2015)

[3] Buitink S. et al., Nature 531, 70 (2016)

[4] Van Haarlem M. P. et al., A\&A 556, 53 (2013)

[5] Thoudam S. et al., Nucl. Instr. Meth. Phys. Res. A 767, 339 (2014) 